\title{
La théorie sociologique générale comme système hiérarchisé de modèles de portée intermédiaire
}

Alban Bouvier

\section{(2) OpenEdition}

\section{Journals}

Édition électronique

URL : http://journals.openedition.org/ress/171

DOI : 10.4000/ress. 171

ISSN : $1663-4446$

Éditeur

Librairie Droz

Édition imprimée

Date de publication : 1 février 2008

Pagination : 87-106

ISBN : $978-2-600-01221-8$

ISSN : 0048-8046

Référence électronique

Alban Bouvier, "La théorie sociologique générale comme système hiérarchisé de modèles de portée intermédiaire ", Revue européenne des sciences sociales [En ligne], XLVI-140 | 2008, mis en ligne le 01 février 2011, consulté le 19 avril 2019. URL : http://journals.openedition.org/ress/171 ; DOI : 10.4000/ ress. 171 
Alban BOUVIER

\section{LA THÉORIE SOCIOLOGIQUE GÉNÉRALE COMME SYSTÈME HIÉRARCHISÉ DE MODÈLES DE PORTÉE INTERMÉDIAIRE}

\section{INTRODUCTION}

Le but de cet article est de ré-examiner le jugement de Robert Merton (1948) - jugement aujourd'hui réactualisé par un certain nombre de chercheurs de premier plan en sciences sociales - selon lequel l'élaboration d'une théorie générale en sociologie est un projet peu pertinent. On juge en effet souvent plus pertinent, dans ce qu'on pourrait appeler aujourd'hui la littérature néo-mertonienne, de s'engager plus que jamais dans la recherche de théories de «moyenne » portée ou de portée «intermédiaire ( (middle-range theories), dont le niveau d'abstraction est par définition moins élevé et la portée en conséquence moins étendue (Hedström et Swedberg, 1998) ${ }^{1}$. On donne toutefois aujourd'hui à la recherche de ces théories, qu'il faudrait probablement plutôt appeler des «modèles » pour s'accorder aux usages épistémologiques actuellement dominants, une tournure qui creuse beaucoup plus profondément les sentiers mertoniens en traversant, de W. Dray (1957) à Nancy Cartwright (1983) et de Raymond Boudon (1984) à Jon Elster $(1998,1999)$, les diverses strates de la critique du modèle nomologicodéductif de l'explication scientifique de Carl Hempel (1942), critiques auxquelles, par nécessité purement chronologique, Merton ne pouvait à cette époque être qu'étranger

Merton (1948) visait explicitement la sociologie de Talcott Parsons; mais des critiques du même type pourraient être formulées (van den Berg, 1998) contre tous ceux qui, d'une manière ou d'une autre, se réclament encore explicitement du modèle parsonien (Habermas, Alexander) ou en sont implicitement tributaires dans leur contestation même (Giddens), voire, pour ne rien lui devoir, ont construit une théorie qui présente certains défauts analogues, tels que la fausse abstraction et donc la fausse généralité ou d'autres défauts, tels le manque d'analyticité (c'est-à-dire l'insuffisante décomposition explicite des concepts problématiques), le verbalisme ou le fréquent excès rhétorique (Bourdieu) ou la tendance au monolithisme explicatif (Bourdieu encore) $)^{2}$. La question reste toutefois de savoir si une théorie générale qui unifierait les théories de moyenne portée

1 Voir aussi Barbera, 2004, Hedström, P., 2005, Edling et Hedström, 2005, Noguera, 2006. Curieusement, aucun des auteurs du volume récemment dirigé par A. Caillé (2004), pourtant consacré à la question de l'idée de théorie générale en sociologie, ne fait référence à la notion mertonienne de middle-range theory.

2 Van den Berg (1998) vise le concept d'habitus et celui de champ qui lui est lié. 
sans présenter les défauts des théories précédentes n'aurait pas une forme de légitimité. Cette question avait été posée par Merton lui-même. Celui-ci n'avait, en effet, nullement renoncé à l'idée d'une unification des «méso-théories » en une macro-théorie sociologique, tâche qui devrait effectivement s'imposer a priori à tout esprit soucieux de donner une interprétation cohérente du monde social (Merton, 1965). Mais Merton avait laissé tout à fait indéterminée la forme que devrait prendre dans le détail une telle théorie, au-delà du choix d'un cadre fonctionnaliste.

Parmi les «néo-mertoniens» notoires, seul James Coleman a cherché à réaliser effectivement un tel programme unificateur, en proposant les Foundations of Social Theory (1990). Considérant qu'il est plus cumulatif de réfléchir à partir d'entreprises déjà existantes que de vouloir tout reprendre ex nihilo, j'examinerai ici de façon préférentielle l'entreprise colemanienne, en tirant partie de façon substantielle de l'oeuvre de deux autres néo-mertoniens d'envergure: R. Boudon (1984, 2003, 2004), qui avait donné implicitement les linéaments suggestifs d'une théorie sociologique cohérente dans La logique du social (1979), sans cependant jamais la développer et J. Elster, foncièrement sceptique, quant à lui, à l'égard des théories générales (Elster, 1990, pp. 12-13).

Si les Foundations ont été, sans surprise, l'objet de nombreuses attaques de divers côtés, le moins que l'on puisse dire est qu'elles n'ont guère été saluées avec enthousiasme par les néo-mertoniens eux-mêmes; elles sont ainsi à peine évoquées dans le volume de Hedström et Swedberg (1998) ${ }^{3}$. Pourtant Coleman est considéré, dans le même volume, comme un des auteurs-phares dans l'élaboration de middle-range theories. A mon sens, le reproche le plus général que l'on peut faire à Coleman est que celui-ci a semblé sinon ignorer du moins minimiser l'importance d'un élément implicite mais essentiel à l'idée même de middle-range theory, à savoir le pluralisme probablement irréductible des principes explicatifs de la théorie sociologique, un pluralisme requis par la très grande diversité des phénomènes sociaux, tels du moins qu'ils sont aujourd'hui accessibles à l'examen. Autrement dit, Coleman se serait rendu lui-même coupable du reproche de tendre au monolithisme explicatif ${ }^{4}$. Un de mes buts, dans cet essai, est de suggérer les manières d'y remédier. On verra, chemin faisant, que Coleman n'évite pas absolument lui-même le reproche sinon d'abus rhétorique du moins de paralogisme argumentatif.

L'idée d'un projet unificateur vaut donc d'être soigneusement réexaminée et d'autant plus que, si la méfiance subsiste aujourd'hui de façon légitime, bien audelà de l'héritage proprement mertonien, à l'égard des théories pseudo-générales, d'un autre côté, peu contestent qu'il y a un dommage considérable à ce que la

3 Pour des réserves explicites ciblées de la part de plusieurs «néo-mertoniens », voir le recueil d'articles publié sous la direction d'A. Bouvier et Ph. Steiner (2003), qui contient notamment des articles de Boudon (2003b), Cherkaoui (2003), Elster (2003), Lazega (2003) et Lindenberg (2003). Voir aussi les critiques de Swedberg (1996) et celles du néo-parsonien Favell (1996).

4 Le ton varie, en réalité, tout au long des Foundations. Parfois, Coleman est très fermé, excluant radicalement les hypothèses alternatives (par exemple, pp. 483-4, à propos du pouvoir explicatif des théories de la frustration relative) - c'est le ton général de l'ouvrage - et parfois il est beaucoup plus ouvert (par exemple, pp. 13-15), comme il l'était, paraît-il, dans les séminaires de Chicago (Elster, 2003, p. 297). 
sociologie soit aujourd'hui aussi fragmentée en de multiples domaines de spécialité et niveaux d'analyse (Caillé, 2004, Dubet, 2004, Kalberg, 2004), ce que la synthèse parsonienne avait sinon réellement évité (la fausse abstraction des multiples étagements de systèmes et sous-systèmes ne pouvant évidemment tenir lieu d'unification véritable) du moins limité en maintenant constamment présent à l'esprit l'idéal d'une discipline unifiée, alors que le précepte mertonien invitant à se concentrer sur la recherche de simples middle-range theories est parfois accusé, tout au contraire, d'avoir participé directement à la fragmentation réelle de la sociologie (Turner, 1991, p. 81).

Je me propose, dans la suite de cet essai, de commencer par réexaminer la notion de middle-range theory telle qu'elle peut être réinterprétée aujourd'hui, i.e. comme modèle analytique de portée intermédiaire de mécanismes générateurs ${ }^{5}$. Dans ce cadre, j'examinerai, en un premier temps, le contexte épistémologique général de cette réinterprétation, marqué par les critiques dirigées contre le modèle nomologico-déductif de Hempel, puis, en un deuxième temps, je montrerai la pertinence d'une distinction entre deux types de mécanismes («intentionnels » $v s$ «non intentionnels »), dont l'analyse est respectivement mise en oeuvre tantôt plutôt par Boudon et tantôt plutôt par Elster. Les deux parties suivantes seront consacrées à la forme que pourrait prendre une théorie sociologique générale. Le troisième temps de cet essai consistera ainsi à mettre en évidence comment les Foundations de Coleman proposent de facto un système, quoique partiel, de modèles de portée intermédiaire de plus ou moins grande abstraction, oscillant entre «intentionnalisme» et «non intentionnalisme». Et le quatrième et dernier temps à montrer comment il est possible d'approfondir et élargir le système colemanien en suivant l'une ou l'autre voies.

\section{I. - MODÈLES ANALYTIQUES (DE PORTÉE INTERMÉDIAIRE) DES «MÉCANISMES GÉNÉRATEURS»: LE CADRE ÉPISTÉMOLOGIQUE GÉNÉRAL}

Le premier mot d'ordre de tous les théoriciens des sciences sociales qui se reconnaissent dans l'esprit néo-mertonien, c'est celui de modéliser analytiquement des phénomènes étroitement circonscrits ; ce qui requiert que, tout en maintenant l'exigence de se situer à un certain niveau d'abstraction pour se distancer de la simple analyse du singulier, on ne s'élève toutefois pas trop haut, pour ne pas perdre tout contact avec le réel empirique. C'est en cela que la modélisation doit se situer à un niveau intermédiaire et la raison pour laquelle, en un premier temps du moins, la question de l'élaboration d'une théorie générale doit être mise entre parenthèses. La question de savoir quelle est la «bonne distance» entre l'abstraction et le réel n'est d'ailleurs guère résolue, dans le contexte mertonien et néomertonien, autrement que par la présentation d'exemples jugés paradigmatiques -

5 Hedström et Swedberg (1998b) considèrent que Merton (1967) et Boudon (1991) constituent les deux meilleures introductions à la «middle-range sociology» (n.3, p.1). 
comme celui de la prédiction créatrice de $\operatorname{Merton}^{6}$ - et non par la détermination de critères de reconnaissance, ce qui serait, en principe, souhaitable mais n'est pas de la première urgence ${ }^{7}$.

Ce qui est visé essentiellement par des auteurs comme Raymond Boudon (1973, 1984) et Jon Elster $(1998,1999)$ - à ma connaissance Coleman ne s'est jamais exprimé explicitement sur cette question - ce n'est pas seulement la défense de la modélisation, en l'occurrence celle de phénomènes bien circonscrits et donc à certains égards locaux (à la différence de l'ambition des théories totalisantes), mais plus précisément la défense de la modélisation de mécanismes, i.e. de processus causaux, par opposition à la simple modélisation de situations ou d'états de choses (modélisation qui est elle-même possible et nécessaire, ce que tendent à minimiser, au demeurant, plusieurs des auteurs du volume précité, identifiant abusivement description et analyse du singulier $)^{8}$. Les premiers, en effet, à la différence des seconds, permettent de donner des explications d'autres phénomènes sociaux: les phénomènes sociaux désignés dans l'explanans (la prédiction qu'il y aura un krach boursier, par exemple) sont invoqués comme mécanismes générateurs des phénomènes sociaux désignés dans l'explanandum (un krach boursier effectif alors que la situation boursière antérieure à la prédiction était saine).

Mais la préoccupation proprement «mertonienne » croise un problème qui en est logiquement indépendant, mais que pose par définition toute théorie explicative $^{9}$. Il s'agit de reprendre la question de savoir s'il est possible de dégager des lois sociologiques, économiques, historiques, etc., question qui a été amenée par Carl Hempel au centre des débats d'épistémologie générale de la seconde moitié $\mathrm{du} \mathrm{XX}^{\mathrm{e}}$ siècle. Le contexte du débat a toutefois considérablement évolué depuis l'élaboration par Hempel de ce qu'il est convenu d'appeler le modèle déductifnomologique.

Boudon (1984) opposait la recherche des modèles à celle des lois, empruntant ses exemples aux sciences sociales. L'un de ces exemples de modèles - il concernait les mécanismes de diffusion des innovations en général - se trouve être un modèle simplement un peu plus général que le modèle d'un des mécanismes précisément donnés comme typique des mécanismes générateurs par Hedström et Swedberg (1998, pp. 18-19), celui de la diffusion d'un nouveau médicament

6 Il s'agit, comme chacun sait, du processus par lequel, dans certaines circonstances, un événement se réalise du fait même qu'il a été prophétisé, le cas classique étant celui de la rumeur (répandue initialement par un petit nombre de personnes) que les banques sont insolvables; si un nombre important de gens les croient et se précipitent pour retirer leur argent en espérant arriver avant que les banques ne soient insolvables, les banques risquent de devenir réellement insolvables.

7 On remarquera que «intermédiaire» ne veut pas dire «médian»: il peut y avoir des modèles plus ou moins abstraits et généraux et donc, corrélativement, plus ou moins concrets et particuliers.

8 La classification raisonnée, qu'elle soit typologique ou non, est en effet une exigence des sciences sociales.

9 Que Parsons propose, quant à lui, une théorie explicative fonctionnaliste ne nous concerne que très indirectement ici. Je ne m'étends donc pas sur les critiques faites au fonctionnalisme lui-même (Hempel, 1959), ni même sur les critiques mertoniennes spécifiques, internes au fonctionnalisme (Merton, 1949). On peut, en outre, concéder que Parsons s'est lui-même intéressé aux «mécanismes générateurs » (Boudon, 1973, pp. 89-90, Chazel, 2006). 
(Coleman, Katz et Menzel, 1957). Nancy Cartwright (1983) avait donné peu auparavant et tout à fait indépendamment, dans un ouvrage devenu depuis lors un classique, une ampleur considérable à une idée analogue. L'idée essentielle de Cartwright, en effet, est que, même en physique et en biologie, il y a une multitude de faits qui ne sont pas, au moins actuellement, subsumables sous des lois de sorte que l'on ne peut rien prédire à leur propos. Pour reprendre l'exemple pédagogique favori de Cartwright (que j'aménage un peu): si un manuel de jardinage (à supposer qu'il puisse être fondé sur des lois de la botaniques analogue à celles de la mécanique générale) indiquait que les camélias plantés dans un terreau tiède poussent en général correctement, on ne pourrait rien prévoir concernant des camélias plantés malencontreusement, par exemple, dans un terreau encore chaud ni expliquer pourquoi, malgré tout, dans de tels cas, certains camélias fleurissent quand même et d'autres pas. Or Cartwright ajoute que ce dont on a pourtant tout particulièrement besoin, dans toute l'«ingénierie» physique et biologique, c'est précisément de la connaissance de ces «mécanismes générateurs» locaux (ici, trivialement: qu'est-ce qui fait pousser les camélias ?), que l'observation, parfois impromptue (ici: probablement plutôt celle du jardinier expérimenté), peut parfois nous apprendre mais dont il faut accepter qu'ils ne sont pas actuellement subsumables sous des lois quoique, dans un avenir situé à l'infini, ce sera peut-être le cas, de sorte que la connaissance des mécanismes générateurs ne justifiera plus alors un type d'attention particulier. En attendant, la connaissance de ceux-ci est du plus grand intérêt et, moyennant le renoncement provisoire à une science idéale, cette connaissance est parfois accessible: pour prendre un exemple moins trivial que celui du jardinage, c'est sur la connaissance de tels mécanismes qu'est fondée toute l'ingénierie de la conquête de l'espace (la mise sur orbite d'un satellite, l'alunissage d'un module, l'arrimage d'un container à la station spatiale, etc.), ce qu'on peut dériver des lois de l'attraction de Newton étant très insuffisant. Jon Elster $(1998,1999)$ resitue explicitement le débat sur la recherche des mécanismes en sciences sociales dans ce cadre cartwrightien, en mettant ainsi clairement en évidence sa signification épistémologique très générale ${ }^{10}$.

Comme il n'est pas possible de subsumer (au moins actuellement) ces mécanismes générateurs sous des lois, on en tire la conclusion que, de toutes façons, si on construit aujourd'hui une théorie générale, elle ne comportera pas non plus de telles lois. Autrement dit, les analyses de Boudon et de Cartwright-Elster fournissent des arguments généraux d'un type nouveau contre le "préjugé nomologique» (l'idée que toute science doive - ou au moins doive exclusivement et préférentiellement - rechercher des lois), débusqué dans ses grandes lignes, pour ce qui est des sciences sociales, par une tradition qui va de Simmel jusqu'à Aron. Ce qu'apportent Boudon et Elster, c'est l'idée de substituer au projet (strictement) nomologique la recherche de «modèles» et de modèles de «mécanismes générateurs », suggestion qui n'était explicite ni chez Simmel ni chez Aron. On remar-

${ }^{10}$ L'article d'Elster (1998) est repris, avec quelques changements, comme premier chapitre de Elster (1999). Elster (1999), p. 2, fait une brève référence à Boudon (1984), à l'occasion de ce thème précis. Sur le rôle de Boudon (1973) dans l'orientation d'un courant de la recherche sociologique contemporaine vers les mécanismes générateurs, voir Cherkaoui (2000), qui, curieusement, ne mentionne pas Elster. 
quera cependant que, bien avant que le concept épistémologique en soit clairement forgé, ce type de recherche avait été pratiqué de fait, plus ou moins confusément, par les historiens-sociologues, de Tocqueville et Weber à Paul Veyne (Elster, 1990, pp. 10-13, Elster, 1999, p.2).

Un processus comme celui de la prédiction créatrice de Merton est un mécanisme générateur typique, on l'a vu. Hedström et Swedberg (1998) donnent deux autres exemples, celui de la diffusion des innovations de Coleman, Katz et Menzel (1957), également aperçu, et celui des effets de seuil de Granovetter (1978). Dans le premier cas, ce qu'on observe régulièrement au niveau macrosociologique, c'est que les innovations (un nouveau médicament, une nouvelle technique agricole) sont lentement adoptées au début du processus puis que le processus s'accélère soudain pour se ralentir enfin, jusqu'à atteindre une vitesse nulle, d'où la forme sinusoïdale que prend la courbe de diffusion; le mécanisme générateur de ce processus réside dans la présence d'un petit groupe d'individus (des médecins ou, respectivement, des agriculteurs) qui, par leur comportement pionnier, jouent le rôle initial tandis que le déclenchement de l'accélération réside dans le fait qu'un nombre plus important d'individus (d'autres médecins, d'autres agriculteurs) leur accordent une certaine autorité ou constatent eux-mêmes les effets positifs de l'innovation adoptée par les pionniers (le ralentissement jusqu'au point mort s'expliquant par le fait que tous les individus virtuellement concernés par l'innovation sont progressivement touchés par elle et l'ont adoptée). Dans le second cas, ce qu'on observe, c'est que le nombre d'individus qui accomplissent déjà une action (par exemple dîner dans un restaurant, s'apprêter à traverser une rue en dehors d'un passage piéton, etc.) a un effet sur le comportement que sont prêts à adopter d'autres individus (rentrer dans un restaurant, s'agglutiner aux piétons qui s'apprêtent à traverser) mais que le nombre-seuil varie selon les personnes. On a bien affaire là à des modèles, i.e. à des représentations schématiques mais clairement dessinées de la réalité, s'appliquant à de nombreux cas singuliers différents, et mettant en évidence quelque chose qui leur est commun qui n'apparaissait pas comme tel auparavant. Ces modèles sont cependant posés comme étant seulement de moyenne portée, ce qui veut dire, d'un côté, que l'on s'abstient de la prétention de fournir avec eux une explication de tous (ou même à peu près tous) les phénomènes sociaux et que, de l'autre, on reconnaît qu'on ne voit pas d'emblée comment les dériver de principes plus élémentaires qui leur seraient communs; on ne se prononce donc même pas non plus, en conséquence, sur la faisabilité de la chose.

\section{II. - MODÈLES DE MÉCANISMES INTENTIONNELS ET MODÈLES DE MÉCANISMES NON INTENTIONNELS}

Ce cadre très général étant fixé, on peut prolonger l'analyse des mécanismes générateurs dans de nombreuses directions en procédant à une succession de distinctions diverses. On peut remarquer, par exemple, que certains de ces mécanismes sont des mécanismes intrinsèquement sociaux au sens où ils impliquent nécessairement des interactions interindividuelles - c'est le cas de tous les exemples types pris par Hedström et Swedberg (1998) (prédiction créatrice, processus de diffusion sinusoïdale, effet de seuil) - alors que d'autres sont des 
mécanismes psychologiques situés au fondement des phénomènes sociaux - c'est le cas des exemples-types de Jon Elster (qui se subdivisent encore, selon Elster $(1998,1999)$, en deux grandes catégories). On peut remarquer par ailleurs que certains des modèles de mécanismes générateurs expliquent des phénomènes de niveau macro par des phénomènes de niveau micro (la prédiction créatrice, le rôle de «l'autorité» des pionniers dans la diffusion des innovations), d'autres des phénomènes micro par des phénomènes macro ou méso (l'effet de seuil), d'autres des phénomènes micro par des phénomènes micro (la genèse de l'ethos capitaliste à partir de modes de comportements initialement justifiés par le protestantisme, selon Weber, Hedström et Swedberg, 1998b, p.5).

Mais un critère est spécialement pertinent pour notre propos, vu la tournure que James Coleman entend donner à la théorie générale et les postures critiques, de types différents et même quasi-symétriques, que Raymond Boudon et Jon Elster adoptent l'un et l'autre par rapport à l'entreprise colemanienne. Ce critère concerne la question de savoir si les causes du phénomène sont supposées intentionnelles ou non. Mais ce critère lui-même ne prend vraiment sens, me semble-til, que si l'on remonte à une strate du débat concernant le modèle nomologicodéductif antérieure aux critiques de type Cartwright, plus précisément au débat entre Dray et Hempel, Dray (1964) retrouvant contre Hempel (1942) une posture wébérienne, voire diltheyenne, comme Raymond Aron (1989) l'a rappelé (p. 128). Il est étonnant que Boudon ne cite qu'à peine Dray dans ses œuvres et seulement pour s'opposer, semble-t-il, à son «anti-hempélisme» radical (Boudon, 1990, pp. 203-4), car la théorie des bonnes raisons de Boudon - elle-même une modélisation ou au moins une schématisation de mécanismes générateurs - me semble s'éclairer grandement de cette confrontation pour de pas dire être débitrice de certaines des idées essentielles mêmes de Dray ${ }^{11}$. C'est notamment le cas si l'on considère l'intégration par Hempel des critiques de Dray à son propre modèle (Hempel, 1965, 1966), En tout cas, une telle posture (modèle hempélien aménagé, suite aux critiques de Dray), même s'il se peut qu'elle ne soit pas exactement celle de Boudon, me semble reposer sur des arguments fort solides et c'est celle que j'adopte ici.

En deux mots, disons que William Dray (1964) montre que, la plupart du temps, il est impossible d'expliquer un événement historique, par exemple tel ou tel choix de Louis XIV, en le déduisant de lois générales, sauf à forger une multitude de lois de ce genre pour expliquer tous les détails de l'événement, des lois qui seront la plupart du temps ad hoc puisqu'on sera le plus souvent bien incapable de présenter les observations qui les fonderaient empiriquement. En revanche, on peut reconstruire les motivations singulières des acteurs et montrer que celles-ci sont raisonnables, vu la situation dans laquelle l'acteur se trouvait ou, plus exactement, vu la perception qu'il avait de cette situation, i.e. que l'on peut, comme dit Boyer (1994) commentant Dray, reconstruire les «bonnes raisons pour agir de telle manière» (p. 226). Cette analyse rationaliste - ou, comme on dit en ce contexte, «rationnelle»-de Dray est en même temps, comme le remarque encore à juste titre Boyer, une «analyse situationnelle» au sens de Popper (pp. 225-6). Et

${ }^{11}$ Sur la notion de «bonne raison» chez Dray, voir par ex. Boyer, 1994 (p. 213-233) et Nadeau, 2005. Boudon (2003a), malgré son titre, ne contient aucune référence à Dray. 
Boudon (1995) insiste lui-même sur le caractère contextuel des raisons en question. Mais, d'après Hempel seconde manière $(1965,1966)$, le modèle de Dray revient simplement à remplacer les lois de couverture psychologiques (du type: «les dirigeants qui poursuivent des politiques contraires à l'intérêt de leurs sujets deviennent impopulaires », Boyer, 1994, p. 225) par des principes normatifs d'action équivalents (du type, sous leur forme la plus générale: «Dans une situation de type $\mathrm{C}$, tout agent rationnel doit faire $\mathrm{X}$ » et, sous une forme plus particulière, par exemple ici: «Tout dirigeant qui cherche à préserver son pouvoir doit éviter de se rendre impopulaire et, en conséquence, doit éviter d'agir contrairement aux intérêts de ses gouvernés »), de sorte qu'on retrouve l'équivalent des lois de couverture (on devrait ajouter, en s'écartant de la lettre de ce que dit Hempel: énonçant des propriétés dispositionnelles) sous la forme de ces principes intentionnels rationnels.

Ce qui est essentiel pour mon propos dans cette controverse, c'est qu'Hempel montre comment le modèle épistémologique des bonnes raisons (formulé initialement par Dray) complexifie le modèle épistémologique nomologique-déductif (formulé par Hempel) en obligeant à en donner une forme plus générale abstraite (le nomos n'est pas nécessairement un principe de la nature, mais peut être un principe d'action), mais ne contredit pas cette forme plus générale.

Ce qui en revanche, affaiblit réellement le modèle nomologique-déductif, ce sont les critiques précédentes (celles du modèle épistémologique de CartwrightElster ou de Boudon) qui montraient que, de toutes façons, ce qui est accessible en matière de principes particuliers de la nature (si c'est eux que l'on vise plus que des principes d'action), ce sont des modèles de mécanismes et non des lois ${ }^{12}$. Mais ce qu'une analyse de type Hempel deuxième manière (i.e. aménagé par les critique de Dray) ajoute alors - et ce que suggèrent également les analyses de Boudon - c'est que la modélisation des mécanismes générateurs eux-mêmes en termes de bonnes raisons pourrait être aussi pertinente ou même plus pertinente que la modélisation en termes de mécanismes non intentionnels (ce que sont les simples dispositions psychologiques) ${ }^{13}$. De sorte que l'on serait légitimé à faire un partage systématique entre les deux types de mécanismes déjà repérés, i.e. selon que ce sont des causes non intentionnelles ou que ce sont des causes intentionnelles, donc éventuellement des raisons. Il est manifeste ainsi qu'Elster se focalise, quant à lui, sur les premiers (les raisins verts, le fait de prendre ses désirs pour des réalités) alors que Boudon non seulement se focalise mais même se centre exclusivement - par principe méthodologique - sur les seconds. Les différentes formes de rationalité (instrumentale, axiologique, cognitive) que distingue Boudon (1995,2003a) constituent ainsi autant d'éléments de «mécanismes générateurs ».

La délimitation entre les deux types de modélisations peut être affinée. Le mécanisme dit des raisins verts consiste en effet en un processus qu'on observe

${ }^{12}$ Ce qui est préservé du modèle de Hempel à ce niveau et qui est essentiel, c'est l'idée qu'il convient de forger des hypothèses (dont le contenu sera constitué de modèles et non de lois) et que de ces hypothèses on pourra «déduire » (ou, plus généralement, dériver) des propositions empiriquement testables.

${ }^{13}$ La notion de mécanisme doit donc ici être entendue en un sens très large, incluant les processus intentionnels. 
régulièrement et qui consiste en ce qu'un individu désire intensément quelque chose, par exemple des raisins qu'il croit bien mûrs; constatant qu'il ne peut atteindre ceux-ci, il ne se contente pas de se résigner mais se convainc ou prétend se convaincre que les raisins ne sont, en réalité, pas si mûrs qu'il l'avait cru au premier abord, qu'ils sont même éventuellement plutôt assez verts de sorte qu'il ne les désire plus du tout. Elster appelle cela le mécanisme des préférences adaptatives (cas particulier d'inversion des préférences) et y voit un processus irrationnel dans la mesure où il y a une incohérence temporelle entre les préférences et que ce renversement des préférences est dû à un pur et simple déni de réalité. Pourtant la démarche serait rationnelle si les raisins étaient effectivement trop verts, que le désir de les manger reposait sur la fausse croyance du contraire et que, reconnaissant après coup son erreur, l'individu se consolait de son échec à les cueillir (la démarche est rationnelle au sens où la proposition empirique initiale en question est falsifiée et remplacée par une proposition conforme à la réalité empirique, i.e. empiriquement vraie $)^{14}$.

En outre, il y a parfois deux descriptions a priori possibles d'un même processus, sans qu'il soit aisé de savoir lequel a effectivement eu lieu. Ainsi, dans l'exemple même de processus que je viens de considérer comme relevant d'une interprétation rationnelle (et désigné de façon indifférenciée sous la métaphore des raisins verts) ${ }^{15}$, la question reste encore de savoir s'il s'agit bien d'une adaptation réfléchie ou au moins quasi-réfléchie ou bien d'un processus largement inconscient, ici tout aussi rationnel, mais d'une rationalité due à une sorte de tendance de l'esprit humain à «réduire les dissonances» (Festinger) en vue de s'adapter au mieux aux circonstances ${ }^{16}$. Il n'est pas aisé de savoir, en dehors de situations expérimentales (et encore...), laquelle est la plus exacte empiriquement. Mais il est raisonnable de penser, en revanche, que lorsque le changement de préférence suppose un déni d'une croyance pourtant fondée, ce processus irrationnel - est inconscient ${ }^{17}$.

Sans vouloir trop anticiper sur la suite de cet essai, on peut déjà chercher à établir une hiérarchie méthodologique provisoire dans l'investigation de ces deux types de mécanismes. Boudon donne ainsi, par méthodologie, une modélisation de l'homo sociologicus plus rationaliste qu'Elster, mais en un sens de «rationaliste » qui reste à préciser. Il conviendra ensuite d'examiner les raisons éventuelles de préférer une méthodologie rationaliste en ce sens-là.

${ }^{14}$ Elster (1986c, pp. 50-2) a proposé de reconstruire ainsi certains des processus décrits par Bourdieu sous le terme d'habitus (Boudon, 1973, pp. 90-2 cite l'habitus comme exemple de mécanisme générateur), par exemple celui par lequel des élèves du secondaire de milieu modeste, désirant a priori faire des études supérieures, s'aperçoivent qu'ils ne le pourront pas et se convainquent qu'en fait ils ne l'avaient jamais vraiment voulu ou voulu seulement parce qu'ils se faisaient une fausse idée de ce que leur donneraient ces études en termes de débouchés. Bourdieu (1987) a contesté cette reconstruction en termes encore trop intentionnels, sinon trop rationnels (p. 20-22).

${ }^{15}$ Voir aussi les différents mécanismes désignés sous le même vocable de «faiblesse de la volonté » (par exemple, in Elster, 1986c).

${ }^{16}$ On pourrait proposer cette interprétation de l'habitus bourdieusien.

${ }^{17}$ Il est certainement possible aussi de redessiner les modèles de l'effet de seuil, de la prédiction créatrice ou de diffusion des innovations dans un cadre rationaliste. Je m'en abstiens ici faute de place. 
Là encore, le postulat de rationalité qu'applique Boudon se comprend mieux et si ce n'est pas non plus exactement la conception dans laquelle se retrouverait Boudon, c'est la conception du postulat de rationalité que je suis enclin à défendre personnellement - si on l'éclaire par les débats épistémologiques qui sont apparus au sein de l'école d'économie austro-américaine, i.e. lorsque von Mises (émigré aux USA) et ses élèves se sont trouvés confrontés aux objections de leurs compatriotes positivistes empiriques du Cercle de Vienne (ou à celles des poppériens) eux-mêmes émigrés ${ }^{18}$. Rizzo (1990) montre, en utilisant les concepts de l'épistémologie de Lakatos, qu'il est épistémologiquement raisonnable de considérer le principe selon lequel les actions sont rationnelles comme le «noyau » dur et intangible du programme de recherche misien ou néo-misien (renoncer à ce noyau, c'est renoncer au programme), tandis que sa «ceinture de protection»-laquelle est destinée à absorber les démentis qu'apporte l'expérience et est, en conséquence, relativement flexible - réside dans le contenu de sens du concept de rationalité. Concession décisive aux objections empiristes, on accepte donc l'idée que le principe de rationalité - compris en tel sens donné - soit falsifiable. Concrètement parlant, cela veut dire que si une action apparaît irrationnelle rapportée à la rationalité conçue comme instrumentale, alors il faut examiner, par exemple, si l'action n'est pas rationnelle par rapport à des valeurs ou bien distinguer entre ce qui était objectivement instrumental et ce qui a paru subjectivement instrumental, et se rabattre par exemple sur la rationalité subjective si la rationalité objective est démentie, etc. Mais ce qui précède ne relève évidemment que d'une stratégie méthodologique: il se peut qu'au bout du compte, les individus dénient parfois irrationnellement la réalité et on devrait être en mesure alors de le reconnaître et même de faire l'inventaire de ces $\operatorname{cas}^{19}$.

\section{III. - LES FOUNDATIONS OF SOCIAL THEORY DE JAMES COLEMAN : DE FACTO, UN SYSTÈME PARTIEL DE MODÈLES DE PORTÉE INTERMÉDIAIRE}

Après avoir examiné en quoi consistait la recherche de modèles intermédiaires de mécanismes sociaux, et indiqué deux grandes façons de hiérarchiser leur analyse, mon propos sera de montrer ici que Coleman reste lui-même jusqu'à un certain point fidèle à l'idée mertonienne d'une recherche de modèles de portée intermédiaire et que les Foundations en regorgent. Coleman ne se contente pas de reprendre la liste de ceux qui ont déjà été découverts ni même d'ordonner celle-ci - ce qu'il fait, au demeurant - mais il allonge en outre cette liste en révélant luimême d'autres modèles de mécanismes générateurs (ce qui justifie donc mal le silence des auteurs du volume de Hedström et Swedberg sur les Foundations). Mais Coleman fait encore plus puisqu'il cherche à établir des liens «horizontaux »

${ }^{18}$ A ma connaissance, Boudon ne s'est démarqué que du von Mises de l'époque viennoise (Boudon, 2004, p. 297), fermé à toute idée de test empirique du principe de rationalité, la rationalité étant alors conçue par Mises comme incluse dans la définition même de l'action.

19 Autrement dit, Boudon est beaucoup plus près de la démarche autrichienne de l'école de von Mises (sinon de celle de celui-ci précisément) qu'il ne semble prêt à l'admettre. 
entre ces modèles (i.e. des liens situés au même niveau d'abstraction), montrant, par exemple, comment on peut transformer un modèle (par exemple le modèle du contrôle d'un individu sur un autre individu) en un autre (par exemple le modèle de l'autorité), moyennant l'introduction d'un tiers terme (en l'occurrence la notion de légitimité) $;{ }^{20}$ puisqu'il cherche, en outre et surtout, à établir des liens «verticaux» entre ces modèles (i.e. des liens de degrés différents d'abstraction), et donc à les hiérarchiser en remontant de modèles de portée intermédiaire, d'un degré d'abstraction inférieur, à des modèles de portée encore intermédiaire mais néanmoins d'un degré d'abstraction supérieur et donc de portée elle-même un peu supérieure, reprenant cette fois le second versant de la pense mertonienne, l'exigence d'une macro-théorie.

Cependant, contrairement à ce que laisse entendre Coleman, sinon toujours du moins souvent, il ne s'agit pas encore d'une théorie générale ou, plus précisément, d'un «système » général de ces modèles - i.e. d'une articulation logique et hiérarchisée complète des modèles ayant montré leur pertinence et, parmi eux, des modèles empiriquement validés - ce que devrait être, à mon sens, une théorie générale analytique. Coleman, en effet, est très illégitimement exclusif dans l'inventaire des modèles pertinents empiriquement validés, alors qu'il est nettement plus généreux dans l'inventaire des modèles disponibles a priori pertinents ${ }^{21}$. Coleman, en tendant même à ériger un des modèles initiaux, a priori de portée intermédiaire (le modèle de la rationalité instrumentale mise au service du selfinterest matériel), en quasi-unique modèle général sans avoir des arguments empiriques suffisamment puissants pour le prétendre, tend du même coup au monolithisme injustifié. Mais il est possible, en éliminant ces paralogismes de la démarche, en rectifiant et en approfondissant les principes mêmes de celle-ci, en faisant apparaître en outre des modélisations alternatives (selon les cas, de type plutôt «intentionalistes» ou plutôt «non intentionaliste»), d'élargir d'une façon épistémologiquement saine le système des Foundations. C'est ce que je me propose d'accomplir sur quelques exemples dans le reste de cette contribution. Prolongeant les analyses précédentes, je centrerai constamment mon propos sur le fait, à mon sens remarquable, que l'on peut donner à la théorie générale une tournure qui l'oriente soit davantage du côté des mécanismes non spécialement intentionnels (à la manière elstérienne) soit davantage du côté des mécanismes intentionnels (à la manière boudonienne), Coleman lui-même semblant hésiter de fait entre les deux tournures.

La démarche «réductionniste» en tant que telle (i.e. l'idée de remonter à du plus général) n'est pas isolée chez les néo-mertoniens et Hedström et Swedberg (1998b) ont cherché eux-mêmes à montrer comment les trois exemples de mécanismes qu'ils avaient choisis comme exemples-types pouvaient être considérés comme des expressions particulières différentes d'un même mécanisme plus

${ }^{20}$ La relation d'autorité de $\mathrm{x}$ sur y (par exemple d'un employeur sur un employé) dérive d'un transfert légitime partiel de contrôle de y sur ses actions à $\mathrm{x}$.

${ }^{21}$ Voir, par exemple, son magistral inventaire des différentes variantes de la théorie de la frustration relative, qu'il va pourtant rayer d'un trait (et avec précipitation) de l'arsenal théorique des modèles empiriquement validés (Coleman, 1990, pp. 472-479). 
abstrait et donc d'application plus générale. Ce noyau résiderait dans la croyance, chez les acteurs sociaux, que le nombre d'individus qui accomplissent un acte est le signe de la valeur probable de l'acte, voire de la nécessité impérative qu'il y a l'accomplir et dans le fait que ce signal a effectivement une influence sur la manière d'agir des individus. Dans le mécanisme de la prédiction créatrice, en effet, le nombre d'individus qui croient initialement à l'énoncé catastrophique est capital, de même que dans celui des processus de diffusion, et l'importance de ce nombre apparaît encore plus clairement dans l'effet dit de seuil. Mais Hedström et Swedberg n'ont pas été plus loin dans leur décomposition-réduction ni n'ont considéré d'autres exemples sur le même modèle (p. 19-21).

Coleman se livre, en revanche, à une "généalogie» beaucoup plus étendue et beaucoup plus unifiée de ces mêmes mécanismes, commençant par rapporter ceux-ci à un même phénomène d'autorité et celui-ci à un «transfert légitime de contrôle». Or bien d'autres phénomènes relèvent, à leur tour, de ce processus très général $^{22}$. Ainsi, Coleman explique les paniques en général, et non pas seulement celles qui sont engendrées par une prédiction «créatrice», par des transferts de contrôle; de même encore les mouvements de foule, les rumeurs, les effets de mode, etc. (Coleman, 1990, pp. 197-240). Mais le même processus entre encore dans l'explication du fonctionnement de la bureaucratie et des entreprises, dans la mesure où celles-ci reposent sur un mécanisme de soumission rationnelle à l'autorité. En procédant à cette réduction par abstraction, Coleman est conscient de lutter contre le morcellement de la sociologie puisqu'il montre comment on peut rattacher à un même modèle général, qui tient lieu d'élément atomique (qui, pour être encore, si l'on veut, de portée intermédiaire par rapport à un unique principe explicatif, est doté d'une portée fort considérable et se distingue à peine d'un modèle général unique), des pans entiers de la sociologie ${ }^{23}$. Mais ce qu'il faudrait ajouter, c'est que ce sont pourtant bien plutôt les maillons intermédiaires entre le ou les modèles abstraits atomiques et la réalité concrète qui sont capitaux dans l'explication: ce qui est explicatif, c'est le plus souvent le composé de divers mécanismes plus élémentaires, donc quelque chose de moléculaire ou de macromoléculaire, non les mécanismes élémentaires, en tant que tels plutôt triviaux et dont la force heuristique isolée reste sujette à caution. Par exemple, ici, ce qui a valeur explicative, c'est la conjonction, outre le rôle de l'autorité, du rôle du nombre d'individus et du rôle de l'effet de signal. Ce que recouvre dans un autre langage l'idée de préférer la recherche de modèles de portée intermédiaire à celle de modèles trop généraux et donc trop abstraits.

L'analyse de Coleman, par ailleurs, n'est manifestement pas toujours exacte, mais il est aisé de la rectifier et de compléter, en conséquence, l'ensemble. Ainsi, dans le cas en question, on peut dire qu'il s'agit plus précisément d'autorité épistémique (Goldman, 1999) - laquelle doit être différenciée de l'autorité politique ou administrative. En effet, dans les trois exemples types, le processus (ou au moins l'un des processus déterminants) consiste dans le fait qu'à certains individus il est reconnu (éventuellement entièrement à tort comme dans le cas de la

${ }^{22}$ Cf. Coleman 1990, p. 226, n.13, à propos de l'effet de seuil de Granovetter. Il est très étonnant que Hedström et Swedberg ne disent pas un mot de cette réduction colemanienne.

${ }^{23}$ Voir notamment Coleman, 1990, p. 197. 
prédiction créatrice) une compétence ou un savoir supérieur: par exemple, dans le cas de l'exemple du restaurant, on suppose que les riverains ont, en général, une certaine connaissance des restaurants qui sont bons et de ceux qui ne le sont pas, de sorte que s'ils s'agglutinent dans l'un, c'est que vraisemblablement la nourriture et le service y sont de qualité, alors que s'il n'y a personne ou presque personne dans l'autre, c'est vraisemblablement que c'est l'inverse. Coleman aurait pu expliquer de la même manière les comportements conformistes déjà modélisés par Homans - mais à un degré d'abstraction plus faible -, le comportement de loyalty (soumission à l'autorité légitime) de Hirschman également, et même celui de voice (comme «révocation» de l'autorité) ${ }^{24}$.

Parfois Coleman recherche la rationalité intentionnelle des comportements, par exemple de la soumission à l'autorité, que ce soit dans des organisations structurées ou que ce soit dans des aspects de la vie sociale aussi informels que l'émergence de paniques collectives. Il lui arrive de pécher alors, du reste, par excès de rationalisme a priori lorsqu'il exclut d'emblée que les mêmes processus (ou plutôt des processus fort voisins) pourraient s'expliquer par des processus inintentionnels et inconscients, ou lorsqu'il en marginalise l'examen. La question se pose pourtant dans le cas des comportements de conformisme ou de soumission à l'autorité $^{25}$.

D'un autre côté, il n'y a rien qui ressemble chez Coleman à la reconnaissance du mécanisme psychologique de la rationalité cognitive que Boudon met spécialement en exergue. Coleman invoque seulement, en effet - comme Elster - l'idée de rationalité limitée quand les comportements ne sont pas rationnels au sens de la rationalité instrumentale par rapport au self-interest matériel ou au sens de la rationalité comme cohérence des préférences entre elles. Ainsi, dans les cas d'incohérences entre les préférences, Coleman préfère supposer un autre type de mécanisme (Coleman, 1990, pp. 504-507) - lequel renvoie à la pluralité du moi (ce que certains auteurs appellent le multiple self (Elster, 1986a)) - de sorte que la rationalité au sens de la cohérence entre les différentes parties du self de l'individu doive finalement être pensée sur le modèle des choix collectifs auxquels se trouvent confrontés un ensemble d'individus (Coleman, 1990, pp. 503-528).

Le choix méthodologique colemanien fait donc clairement sortir du cadre de l'individualisme méthodologique pour descendre à un niveau d'explication encore plus bas ( «infra-individualiste») et y chercher de nouveaux «mécanismes générateurs », lesquels supporteraient apparemment assez bien d'être modélisés eux-mêmes comme des composés des mêmes types d'atomes primitifs: la recherche du «self »-interest d'une partie du self (constituant une sorte de microself) s'opposant à une autre partie du «self» (autre micro-self) et cherchant à le

${ }^{24} \mathrm{Si}$ Coleman ne dit rien du modèle de Homans, on pourra comparer, en revanche, la réduction effective que propose Coleman du modèle de Hirschman (Foundations, pp. 457-9).

${ }_{25}$ Ainsi, curieusement, Coleman ne dit pas un mot des expériences pourtant célèbres de Milgram. Et s'il évoque (rapidement) celles de Asch et s'il ne remet aucunement en cause leur portée - puisqu'il ne prétend même pas dire plus que: «dans certains cas au moins [les phénomènes en question] peuvent être expliqués par les principes du choix rationnel [souligné par moi]» (p. 288) - il ne leur accorde pourtant aucune considération supplémentaire dans les Foundations. 
contrôler $^{26}$. La vue est peut-être profonde mais n'implique pas, comme le montrent en effet a contrario les propres propositions boudoniennes (qui conservent l'hypothèse de rationalité individuelle en faisant varier le contenu du concept de rationalité), qu'aient été épuisés les mécanismes de niveau individualiste. On pourrait pourtant a priori invoquer aussi, plus sobrement, dans le cas de certaines inversions de préférences, l'apparition de raisons plus fortes de choisir dans le sens opposé (explication cognitive de type boudonien). L'analyse de Coleman suggère malgré tout qu'une théorie générale qui intégrerait les mécanismes de type non intentionnel pourrait finir par devoir descendre au niveau infra-individualiste (p. 14 et p. 505, avec référence au mécanisme de la faiblesse de la volonté). Mais Coleman va en réalité cette fois bien au-delà d'Elster car Elster (1986b) lui-même entend, au contraire, épuiser bien plus que Coleman les ressources de l'individualisme méthodologique dans le cadre même d'une perspective non intentionnaliste.

\section{IV. - PERFECTIONNEMENTS ET ÉLARGISSEMENTS DES FOUNDATIONS. VOIE «INTENTIONALISTE» ET VOIE «NON INTENTIONALISTE»}

La théorie de Coleman est donc aménageable, et on a déjà perçu en quels termes. Ce qu'il s'agirait de faire, c'est de proposer une théorie plus intégrante que celle de Coleman et donc plus générale qu'elle ne l'est effectivement au sens où les modèles proposés puis retenus comme pertinents seraient en nombre plus nombreux et porteraient en conséquence sur davantage de cas, et pas au sens (colemanien) où l'un des modèles ou quelques-uns d'entre eux seraient supposés rendre compte à eux seuls de l'ensemble des phénomènes sociaux. L'une des extensions possibles inclurait la prise en compte systématique des mécanismes tant de type intentionnel que de type non intentionnel. On a vu comment Coleman hésitait, au contraire, entre l'un et l'autre axes. Je me propose d'envisager très rapidement, dans la suite de cette contribution, quelques-uns des prolongements possibles de la théorie colemanienne.

Pour bien mesurer ce qui est en jeu, il convient de prendre un peu de recul et considérer la structure d'ensemble des Foundations. On fait souvent de la théorie colemanienne une expression particulièrement typée de la Théorie du Choix Rationnel $(\mathrm{TCR})^{27}$. Mais c'est loin d'être exact et on s'en aperçoit aisément lorsque l'on considère le mode de progression principal (l'axe directeur) des Foundations. Ce n'est pas du tout, en effet, un mode de progression par extension de la notion de rationalité comme dans le programme «néo-misien» (au sens défini) que Boudon développe depuis L'Art de se persuader, mais un mode de

${ }^{26}$ Cet infra-individualisme est implicite dès le premier chapitre des Foundations (cf. pp. 13-15) et explicite au chapitre 19 (p. 505), au travers de la référence aux psychologues sociaux Thaler et Shefrin (1981), même si le terme lui-même est absent, référence qui permet de se convaincre que la construction conceptuelle colemanienne n'est pas purement verbale.

27 Boudon (2003a et b) lui-même. Golthorpe (2000) fait exception. 
progression dont on devrait plutôt dire qu'il est «néo-smithien». Coleman progresse, en effet, dans le cadre d'un enrichissement progressif (au demeurant, vite interrompu) d'une théorie des relations, spécialement des relations sociales et, plus précisément, dans le cadre d'une théorie des motivations relationnelles. Coleman se donne au début des Foundations deux relations et seulement deux: celle d'intérêt matériel (pour des objets, des personnes, des événements, etc.), celle de contrôle (sur des objets, des personnes, etc.). Mais Coleman introduit ensuite la sympathie au sens de processus d'identification à autrui (c'est le moment proprement smithien (Smith, 1999 [1759]) pour expliquer certains types de soumission à l'autorité ainsi que les comportements authentiquement altruistes (par opposition à l'altruisme calculateur). Il s'agit bien là d'un autre type de mécanisme (psychologique) générateur de phénomènes sociaux. Mais dans toutes les Foundations, les seules notions de rationalité explicitement en jeu (les seuls «mécanismes générateurs psychologiques rationnels»), on l'a vu, ce sont la rationalité instrumentale, limitée elle-même à l'idée d'adaptation à ce qui permet de satisfaire le self-interest matériel, d'un côté, et la rationalité comme cohérence entre les préférences. Les limites de l'une et de l'autre (la notion de rationalité limitée est donc assumée) sont résolues, dans le premier cas, par l'introduction de la notion de «sympathie» et, dans le second, par le passage à l'infra-individualisme.

Il n'est pas inutile, à ce point de la démarche, de prolonger les analyses colemaniennes en les confrontant à une démarche analogue menée avant lui par un économiste hétérodoxe, Amartya Sen, dans la mesure où cette démarche est également (et tout aussi explicitement) néo-smithienne ou néo-humienne, quoique en un premier temps seulement puisque, en un deuxième temps et tout aussi explicitement, elle est ensuite néo-kantienne ${ }^{28}$. Sen (1993) fait référence à des exemples du même type que ceux de Coleman; comme lui, il met en évidence que certains comportements ne peuvent pas du tout s'expliquer par la seule référence à un self-interest matériel au service duquel se mettrait la rationalité instrumentale. Mais le but de Sen est d'aller encore plus loin et de suggérer qu'un mécanisme qui sollicite un sentiment, tel que la sympathie, n'est pas encore suffisant et qu'un certain nombre de comportements, y compris de comportements qui affectent l'économie - mais évidemment aussi bien d'autres comportements sociaux ne s'expliquent pas bien si on n'invoque pas un processus (un «mécanisme générateur») d'un autre ordre, qu'il appelle l'engagement (commitment) et qui aurait des affinités avec l'impératif kantien ou, de façon générale, avec ce que l'on est supposé faire uniquement par devoir. Ce n'est pas le lieu de discuter la proposition de Sen, quoique celle-ci ait été très controversée, même parmi ceux qui considèrent qu'il est hautement désirable que les comportements économiques suivent une certaine éthique. Mon but est simplement de montrer que, lorsqu'on suit une veine smithienne, on peut être amené à se poser la question du caractère suffisant ou non d'une explication de comportements d'allure morale en invoquant uniquement des sentiments. Il faut cependant convenir que cet aspect de la pensée tant de Coleman que de Sen reste très inchoatif et peu élaboré.

\footnotetext{
${ }^{28}$ Curieusement, là aussi, Coleman ne cite pas Sen sur ce point.
} 
Invoquer des sentiments à la source de mécanismes sociaux, c'est adopter une voie non intentionaliste, dans la mesure où ces sentiments émergent d'une façon qui n'est pas intentionnelle. Pourtant, une voie intentionnaliste existe. Boudon, sans commenter Coleman dans le détail (ni non plus se référer à Sen, dont la position, amplement discutée dans la littérature, est pourtant fort voisine de la sienne), propose en effet une voie alternative pour rendre compte des mêmes phénomènes en termes de mécanismes générateurs, puisqu'il suppose, au contraire, des intentions et des intentions rationnelles ${ }^{29}$. C'est que l'élargissement de la théorie sociologique à partir, comme chez Boudon, d'un point de départ situé dans la rationalité comme adaptation à la fin recherchée mise au service du self-interest matériel procède en suivant non pas la voie des types de motivations mais celle des types de rationalités.

Cependant, au lieu d'introduire ici la notion très spécifique de rationalité axiologique que Raymond Boudon propose (elle suppose, en effet, que les préférences aient une valeur morale objective) et sur laquelle Boudon s'est déjà beaucoup étendu, je souhaite simplement rappeler, pour conclure, l'existence d'autres alternatives plus classiques, que Boudon (2003a, 2004) évoque à peine. Celles-ci sont tout aussi intentionnalistes mais requièrent une notion beaucoup moins forte de rationalité (la rationalité subjective ou transsubjective suffit). Au demeurant, pour évaluer dans quelle mesure l'introduction de la notion de rationalité axiologique au sens de Boudon constitue un élargissement ou un renforcement de l'explication sociologique, il faudrait, de toutes façons, revenir au préalable sur la notion de rationalité instrumentale telle que la conçoivent eux-mêmes Boudon et Coleman. En effet, l'un et l'autre restreignent implicitement le plus souvent la rationalité instrumentale à la rationalité comme adaptation des moyens en vue de satisfaire le self-interest matériel (Coleman, 1990, Boudon, 2003a, 2004), suivant ici l'usage le plus fréquent de la théorie dite du choix rationnel dans les études appliquées (par exemple en sciences politiques, dans la sociologie de l'action collective, etc.).

Mais il est patent que la version de la TCR dont part Coleman (et que Boudon prend comme référence) est très étroite par rapport aux versions disponibles et que celles-ci offrent la possibilité de rendre compte de ce qui apparaît comme des énigmes dans le cadre de cette version étroite, comme le paradoxe du vote (Boudon, 2003a et b). Non seulement cela, mais la version la plus répandue de la TCR depuis que l'idée en a été introduite en économie (i.e. depuis Pareto) est, en son principe, que la rationalité qu'il convient de considérer, c'est - outre la rationalité comme cohérence entre les préférences - la rationalité comme adaptation des moyens aux fins recherchées (les «préférences», les «utilités»), quelle que soit la nature de ces fins, notion qu'on peut ensuite spécifier en introduisant les notions de coût et de bénéfice ou d'avantage et celle de maximisation des avantages et de minimisation des coûts ${ }^{30}$. De sorte que selon cette version plus étendue

${ }^{29}$ Voir notamment Boudon (2003a).

${ }^{30}$ Coleman dit très explicitement que sa théorie suppose seulement, dans les quatre premières parties, que les hommes sont susceptibles de se donner des fins (il sont capables d'une «purposive action»); seule la cinquième et dernière partie ajoute l'idée de maximisation des utilités (Coleman, 1990, p. 18). 
de la TCR, l'altruisme peut fort bien être considéré comme une fin tout comme pourraient l'être son honneur, la libération de son pays, l'instauration d'une république égalitaire - ou, tout aussi bien, la restauration de l'esclavage, celle de la peine de mort, etc..

Rien n'empêche donc d'étendre la notion de rationalité instrumentale ellemême pour qu'elle rende compte de la quête de valeurs ou de fins considérées comme telles: sera rationnelle une action qui sera adaptée à la fin poursuivie; il sera ainsi rationnel par rapport à la restauration de son honneur, dans des sociétés ou celui-ci est valorisé, de rechercher le duel et donc de mettre en danger sa vie ou son intégrité physique, etc. ${ }^{31}$ On pourra alors préférer à l'expression de rationalité instrumentale celle moins connotée de rationalité moyen/fin (ou means/end rationality comme disent les anglo-saxons) $)^{32}$.

On remarquera enfin que la notion de rationalité comme cohérence entre les préférences (ou entre les «fins»), celle que l'on a déjà rencontrée (à propos de la fable des raisins verts) et qui est utilisée en théorie de la décision permet ellemême de rendre compte de comportements qui n'obéissent nullement à la rationalité dans la recherche de son self-interest matériel mais qui sont rationnels sous le rapport de la cohérence avec les choix antérieurs: ainsi, si j’ai préféré dans telle circonstance passée, la préservation de mon honneur à celle de mon intégrité physique, il est rationnel (au sens de cohérent) de le préférer encore aujourd'hui.

\section{CONCLUSION}

Dans cette contribution, j'ai cherché à examiner la forme que devrait prendre, à mon sens, une théorie générale en sciences sociales, en réfléchissant à partir de la réalisation récente d'élaboration d'une telle théorie générale, les Foundations of Social Theory de James Coleman. Une telle théorie doit user d'un style analytique, être rhétoriquement sobre et intégrer les critiques de Robert Merton à l'égard des pseudo-théories générales. Elle doit donc, en conséquence, attirer l'attention sur la recherche de modèles, voire de micro-modèles, des mécanismes de types variés qui seraient à la source des phénomènes sociaux. Ces modèles donnent le plus souvent à voir des mécanismes moléculaires c'est-à-dire des mécanismes formés eux-mêmes de processus plus élémentaires («atomiques») dont les modèles sont par nature peut-être trop abstraits pour avoir, comme tels, un réel pouvoir heuristique. J'ai cherché, d'autre part, à montrer que ces modèles sont essentiellement de deux types, selon qu'ils schématisent des mécanismes intentionnels ou non intentionnels et soutenu qu'une théorie générale devrait intégrer les uns et les autres.

\section{Unité d'Aix-Marseille (Université de Provence)}

${ }^{31}$ Coleman ne fait qu'évoquer ce modèle mais il reconnaît explicitement la pertinence d'un modèle qui ferait de l'honneur la fin visée, notamment pour les sociétés antérieures à la Renaissance occidentale (p. 28, avec référence à Hirschman, 1980).

${ }^{32}$ Wolfelsperger (2001) a recensé un certain nombre de procédures du même ordre permettant d'utiliser la notion de rationalité classique en économie afin de rendre compte des comportements visant des «valeurs» sans introduire pour autant la rationalité axiologique boudonienne. 


\section{BIBLIOGRAPHIE}

Aron, R., 1989, Leçons sur l'histoire, Paris, Ed. de Fallois.

Barbera, F., 2004, Meccanismi Sociali. Elementi di sociologica analitica, Bologna, Il Mulino.

Boudon, R. 1973, L'inégalité des chances. La mobilité sociale dans les sociétés industrielles, Paris, Armand Colin.

Boudon, R., 1979, La logique du social, Paris, PUF.

Boudon, R., 1984, La place du désordre, Paris, PUF.

Boudon, R., 1991, «What Middle-Range Theories Are». Contemporary Sociology, 20, pp. 519-522.

Boudon, R., 1990, L'art de se persuader des idées douteuses, fragiles ou fausses, Paris, Fayard.

Boudon, R., 1995, Le Juste et le Vrai, Paris, Fayard.

Boudon, R., 1998, «Social mechanisms without black boxes » in Hedström, P. et Swedberg, R. (dir.), 1998a, pp. 172-203.

Boudon, R., 2003a, Raison, Bonnes Raisons, Paris, PUF.

Boudon, R., 2003b, «La conversion de Coleman à la théorie du choix rationnel: impressions et conjectures » in A. Bouvier et Ph. Steiner, 2003, pp. 389-398.

Boudon, R., 2004 (2002), «Théorie du choix rationnel ou individualisme sociologique ?» in Revue du MAUSS, $\mathrm{n}^{\circ} 24$, pp. 281-309.

Bourdieu, P., 1987, Choses dites, Paris, Minuit.

Bouvier, A., 2003, «Dans quelle mesure la théorie sociale de James Coleman est-elle trop parcimonieuse?», in A. Bouvier et Ph. Steiner, 2003, pp. 331-356.

Bouvier, A., 2006, «L'architecture de la sociologie», in Revue du MAUSS, n² 28, pp. 391-402.

Bouvier, A. et Steiner, Ph., 2003, A. Bouvier et Ph. Steiner (ed.), La théorie du choix rationnel: les Foundations of Social Theory de James $S$. Coleman en débat ${ }^{\circ}$ spécial de la Revue française de sociologie, 2003, T. 44-2.

Boyer, A., 1994, Introduction à la lecture de Karl Popper, Paris, Presses de 1'Ecole Normale Supérieure.

Caillé, A., 2004, Présentation du numéro spécial de la Revue du MAUSS, $\mathrm{n}^{\circ}$ 24, Une théorie sociologique générale est-elle pensable? De la science sociale,p. 7-44.

Cartwright, N., 1983, How the Laws of Physics Lie, Oxford, O.U.P..

Chazel, F., 2006, «Les étapes de l'abstraction en sociologie: actualités et limites du cheminement parsonien», Année sociologique, Vol. 56, n², pp. 353-368.

Cherkaoui, M., 2000, «La stratégie des mécanismes générateurs comme logique de l'explication» in Baechler, Chazel, Kamrane (dir.) (2000), L'acteur et ses raisons. Mélanges en l'honneur de Raymond Boudon, p.130-151.

Cherkaoui, M., 2003, «Les transitions micro-macro. Limites de la théorie du choix rationnel dans les Foundations of Social Theory», in Bouvier et Steiner, 2003, pp. 231-254.

Clark. J., 1996, James S. Coleman, London, The Falmer Press.

Coleman, J.,1990, Foundations of Social Theory, Cambridge (Mass.): University of Harvard Press.

Coleman J.,E. Katz et H. Menzel, 1970 [1957], «La diffusion des innovations chez les médecins » in Lazarsfeld, Boudon, Chazel (eds).

Dray, W., 1957, Laws and Explanation in History, Oxford, O.U.P.

Dubet, F., 2004, «Pourquoi rester «classique»?» in Revue du MAUSS, n 24, pp. 219-232.

Edling, C. et Hedström, P., 2005, «Analytical Sociology in Tocqueville's Democracy in America», working papers series, on line.

Elster, J. (ed), 1986a, The Multiple Self, Cambridge: Cambridge University Press.

Elster, J., 1986b, «Introduction» à Elster, J., 1986a, The Multiple Self, Cambridge, C.U.P.

Elster, J., 1986c (1983), Le laboureur et ses enfants. Deux essais sur les limites de la rationalité, Paris, Minuit. 
Elster, J., 1989, Nuts and Bolts for the Social Sciences, Cambridge, C.U.P.

Elster, J., 1990, Psychologie politique, Paris, Minuit.

Elster, J., 1998, « A plea for mechanisms », in Hedström, P. et Swedberg, R. (dir.), 1998a, pp. 45-73.

Elster, J., 1999, Alchemies of the Mind, Cambridge, C.U.P.

Favell, A. 1996, «Rational Choice Theory as Grand Theory: James Coleman's Normative Contribution to Social Theory » in Clark (1996).

Elster, J., 2003, «Coleman on Social Norms» in Bouvier et Steiner, 2003, pp. 297-304.

Goldman, A., 1999, Knowledge in a Social World, Oxford, O.U.P.

Goldthorpe, J., 2000, On Sociology. Oxford: Oxford University Press

Granovetter, M., 1978, «Threshold models of collective behaviour », American Journal of Sociology, $\mathrm{n}^{\circ} 66$, pp. 246-258.

Hedström, P., 2005, Dissecting the Social. On the Principles of Analytical Sociology, Cambridge, C.U.P.

Hedström, P. forthcoming, «Rational Choice Theory and Sociology », in L. Blume and S. Durlauf, The New Palgrave Dictionary of Economics, disponible on line.

Hedström, P. et Swedberg, R. (dir.), 1998a, Social Mechanisms. An Analytical Approach to Social Theory,Cambridge, C.U.P.

Hedström, P. et Swedberg, R. (dir.), 1998b, «Social mechanisms. An introductory essay » in Hedström, P. et Swedberg, R. (dir.), 1998a, pp. 1-31.

Hempel, C., 1942, «The Function of General Laws in History» in M. Martin et L.McIntyre, 1994, pp. 43-54.

Hempel, C., 1959 «The Logic of Functional Analysis» in in M. Martin et L.McIntyre, 1994, pp. 349375.

Hempel, C., 1965, Aspects of Scientific Explanation, New York, Free Press.

Hempel, C., 1966 [1962], «Explanation in Science and in History» in W. Dray (ed.), 1966, Philosophical Analysis and History, New York, Harper and Row.

Hirschman, A.O. 1972 (1970), Face au déclins des entreprises et des institutions, Cambridge, C.U.P.

Hirschman, A.O., 1980 (1977) Les passions et les intérêts. Justifications politiques du capitalisme avant son apogée, Paris, P.U.F., 1980.

Kalberg, St., 2004, «Difficultés d'un consensus transnational sur une théorie sociologique unifiée », in Revue du MAUSS, $\mathrm{n}^{\circ}$ 24, pp. 173-188.

Kuran, T., 1998, «Social Mechanisms of Dissonance Reduction» in Hedström et Swedberg, 1998, pp. 147-171.

Lazega, E., 2003, «Rationalité, discipline sociale et structure«, in A. Bouvier et Ph. Steiner, 2003, pp. 331-356.

Lindenberg, S., 2003, «Coleman et la construction des institutions: peut-on négliger la rationalité sociale ?» in A. Bouvier et Ph. Steiner, 2003, pp. 357-373.

Martin, M. et L.McIntyre, 1994, Readings in the Philosophy of Social Science, MIT Press, Cambridge (Mass.)

Merton, R., 1948, «Discussion of Parsons' «The Position of Sociological Theory», American Sociological Review, 13, pp. 164-8.

Merton, R., 1965 (1949), Eléments de théorie et de méthode sociologique, Paris, Plon.

Merton, R., 1967, «On Sociological Theories of the Middle-Range» in On Theoretical Sociology, New York, The Free Press.

Nadeau, R., 2005, «L'explication rationnelle en histoire. Dray, Collingwood et Hempel», in Ch. Nadeau (dir.), Mélanges offerts en l'honneur de Maurice Lagueux, Québec, Presses de l'Université Laval.

Noguera, J.A., 2006, «Why We Need an Analytical Sociological Theory», in Papers, Revista de Sociologia, Barcelona. 
Pettit, Ph., 1998, «Republican Theory and Political Trust», in V. Bratwaite et M. Levi (eds), Trust and Governance, New York, Russell Sage Foundation.

Rizzo, M. 1990, «Mises and Lakatos. A Reformulation of Austrian Methodology », in St. Littlechild (1990), Austrian Economics, vol.1, Edward Elgar, Great Yarmouth., pp. 487-507.

Sen, A., 1993 (1982), «Des idiots rationnels. Critique de la conception du comportement dans la théorie économique » in Sen, 1993, Ethique et économie, Paris, PUF.

Smith, A., 1999 (1759), Théorie des sentiments moraux, Paris, PUF.

Swedberg, R., 1996, «Analyzing the Economy: On the Contribution of James S. Coleman» in Clark (1996), pp. 313-328.

Swedberg, R., 2006, «Quand la sociologie économique rencontre l'économie des conventions », in Eymard-Duvernay, F. (dir.), 2006, L'économie des conventions. Méthodes et résultats. Tome I: Débats, Paris, La Découverte, pp. 77-92.

Thaler, R.H. et H.M. Shefrin, 1981, «An Economic Theory of Self-Control», in Journal of Political Economy, $\mathrm{n}^{\circ} 89$, pp. 392-406.

Turner, J., 1991, 5th ed. ([1974], The Structure of Sociological Theory, Wadsworth Publishing Company, Belmont (Cal.).

Van der Berg, A., 1998, «Is Social Theory too Grand for Social Mechanisms?» in Hedström, P. et Swedberg, R. (dir.), 1998, pp. 204-237.

Wolfelsperger, A., 2001, «La modélisation économique de la rationalité axiologique: des sentiments moraux aux mécanismes sociaux de la moralité » in R. Boudon, P. Demeulenaere et R. Viale, L'explication des normes sociales, Paris, PUF. 